\title{
A Novel Flexible Magnetic Composite Material for RFID, Wearable RF and Bio-monitoring Applications
}

\author{
Li Yang ${ }^{1}$, Lara Martin ${ }^{2}$, Daniela Staiculescu ${ }^{1}$, C. P. Wong ${ }^{1}$, and Manos M. Tentzeris ${ }^{1}$ \\ ${ }^{1}$ Georgia Institute of Technology, Atlanta, GA, 30332, U.S.A. \\ ${ }^{2}$ Motorola, Plantation, FL, 33322, U.S.A. \\ E-mail: liyang@ece.gatech.edu
}

\begin{abstract}
This paper introduces for the first time a novel flexible magnetic composite material for RFID and wearable RF antennas. The successful implementation of the flexible magnetic composite material will enable the significant miniaturization of RF passives and antennas in UHF frequency bands, especially for applications requiring conformal modules that can be easily finetuned. A conformal RFID tag working at $480 \mathrm{MHz}$ is then fabricated and the miniaturization concept proven.
\end{abstract}

Index Terms - Magnetic composites, RFID, UHF, RF passives, relative permittivity, loss tangent

\section{INTRODUCTION}

The inception of RFID (radio frequency identification) has enabled contactless transfer of information without the requirement of line of sight association, specifically between a reader and transponders that reside on identified items. As the technology for RFID systems continuously improves and extends to structures of non-orthogonal shapes and to conformal sensors of wireless body area networks (WBAN), there has been a need to design more "flexible" reader and tag systems. Namely miniaturization of the transponder and ability to tune the system performance to accommodate EM (electromagnetic) absorption and interference from surrounding media, while compensating for fabrication tolerances has been one of the major priorities [1]. Threedimensional transponder antennas that utilize wound coil inductors do make use of magnetic cores, but they are quite bulky and impractical. On the other side, magnetic materials for two-dimensional embedded conformal planar antennas have not yet been successfully realized for standard use. This paper introduces for the first time a novel flexible magnetic composite for printed circuits and antennas, that can reap the same miniaturization and tuning benefits with the heavier and non-flexible 3D used magnetic cores.

One of the most significant challenges for applying new magnetic materials is understanding the interrelationships of the properties of the new materials with design and performance of the specific topology (e.g. radiation pattern, scattering parameters). In previous studies, it can often be cited that the objectives of miniaturization and improved performance are tempered by the limited availability of materials that possess the required magnetic properties, while maintaining an acceptable mechanical and conformality performance [2]. Recently, formulation of nano-size ferrite particles has been reported [3] and formulation of magnetic composites comprised of ferrite filler and organic matrix has been demonstrated [4]. The implication of these new magnetic materials has yet not been investigated for specific EM systems above the low $\mathrm{MHz}$ range. Additionally, in the cases of complex microwave systems involving numerous interconnects, dielectric interfaces or radiating structures, the co-design of the materials along with the structure dimensions and the fabrication design rules may be necessary in order to achieve the optimal targeted performance. The aim of this work is to provide a basis for this co-design of materials, fabrication processes and electromagnetic structures, namely for the benchmarking case of a novel flexible magnetic composite, a BaCo ferrite-silicone composite, and a UHF RFID antenna, respectively.

Specifically, in this study a benchmark structure was first designed for $480 \mathrm{MHz}$ in a full-wave simulator for an unfilled Silicone substrate; then the magnetic nano-particles were added and the same antenna was redesigned for $480 \mathrm{MHz}$ by reducing its size, thus proving the miniaturization concept. The next step was the actual fabrication of the material and the measurement of the electrical and magnetic characteristics, including loss. Finally, the miniaturized antenna was fabricated on the magnetic composite and its performance was measured, compared and validated along with the simulated predictions. The presented magnetic substrate is the first flexible magnetic composite tested and proven for the 480 $\mathrm{MHz}$ bandwidth with acceptable magnetic losses, that makes it usable for lightweight conformal/wearable applications like pharmaceutical industry and wireless health monitoring in hospital, ambulance and home-based patient care.

\section{MATERIAL DEVELOPMENT}

The first step for this work was to develop a magnetic composite that provides the advantage of low temperature processing for compatibility with organic substrate processing, flexibility, and high adhesion. With regard to these three properties, the magnetic composite would have to 
be compatible with common substrates used for RFID, such as polyethylene terephthalate (PET) and polyimide. Additionally, the composite dielectric loss can affect circuit performance, and low dielectric loss would be targeted. For these objectives, properties of candidate matrices would be the same, that is, low temperature processibility, high flexibility, high adhesion, and low dielectric loss. Dielectric constant can also affect the circuit performance and should also be monitored. The matrix materials considered candidates for this proposed work included silicone, UV curable acrylic-based adhesives, and benzocyclobutene (BCB). Silicone provides reasonable viscosities required for good filler mixing during processing, that is, not too low to promote settling and not too high for uniform mixing. Additionally, silicone provides the properties of flexibility and, for some formulations, good adhesion.

After careful analysis, the matrix material choice was made for Dow Corning Sylgard 184 silicone. The electrical parameters of the unfilled silicone, used in the initial antenna design, are $\varepsilon_{r}=2.65$ and $\tan \delta_{e}=0.001$. The choice for the magnetic composite was Trans-Tech BaCo ferrite powder, product name Co2Z. A 40 vol\% ferrite paste was produced with a mixer at $240 \mathrm{rpm}$ and $110^{\circ} \mathrm{C}$ for 30 minutes. The paste was transferred into a flat mold and vacuum cured with a hold confirmed to occur at $>125^{\circ} \mathrm{C}$ for 50 minutes to produce a 1.3 mm thick substrate.

The material was measured using an HP4291A impedance analyzer to obtain complex permittivity $(\varepsilon)$ and permeability $(\mu)$ (real and imaginary parts) with material fixtures 16453A for $\varepsilon$ and 16454A for $\mu$ over the frequency range of $1 \mathrm{MHz}$ to $1.8 \mathrm{GHz}$. There were 5 measurements taken for each $\varepsilon_{r}, \mu_{r}$, $\tan \delta_{e}$ and $\tan \delta_{m}$. The summary statistics, including the mean and $95 \%$ C.I. (confidence intervals) for $\varepsilon_{r}, \mu_{r}, \tan \delta_{e}$ and $\tan \delta_{m}$ of the ferrite composite at $480 \mathrm{MHz}$ are given in TABLE I. Based on these results, the values used in the model were $\varepsilon_{r}=$ $7.14, \mu_{r}=2.46, \tan \delta_{e}=0.0017$ and $\tan \delta_{m}=0.039$.

TABLE I

MEAN AND 95\% CONFIDENCE INTERVALS FOR $\varepsilon$ AND $\mu$ MEASUREMENTS OF FERRITE COMPOSITE AT $480 \mathrm{MHz}$

\begin{tabular}{|c|c|c|c|}
\hline & mean & Lower CI & Upper CI \\
\hline$\varepsilon_{r}$ & 7.142 & 7.083 & 7.201 \\
\hline$\mu_{r}$ & 2.463 & 2.457 & 2.468 \\
\hline $\tan \delta_{e}$ & 0.0017 & 0.0005 & 0.0028 \\
\hline $\tan \delta_{m}$ & 0.0391 & 0.0358 & 0.0424 \\
\hline
\end{tabular}

\section{ANTENna Design and MEAsurement}

The demand for flexible RFID tags has rapidly increased due to the requirements of automatic identification in itemlevel tracking. The UHF RFID band varies in frequency, power levels, number of channel and sideband spurious limits of the RFID readers signal, depending on the application and

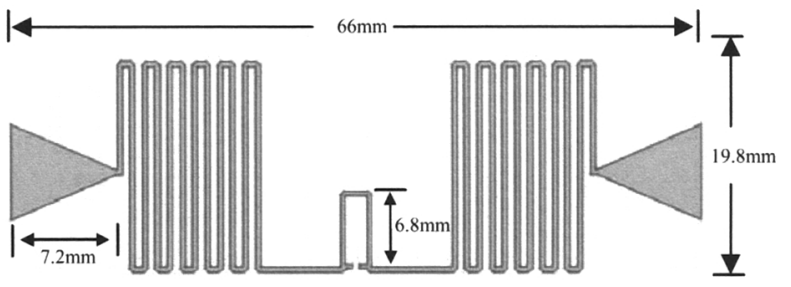

Fig. 1. Configuration of the RFID tag module on magnetic composite substrate.

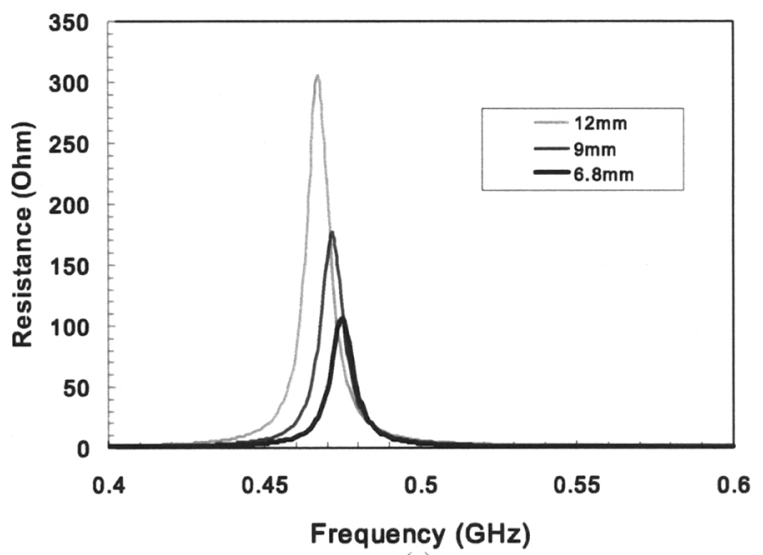

(a)

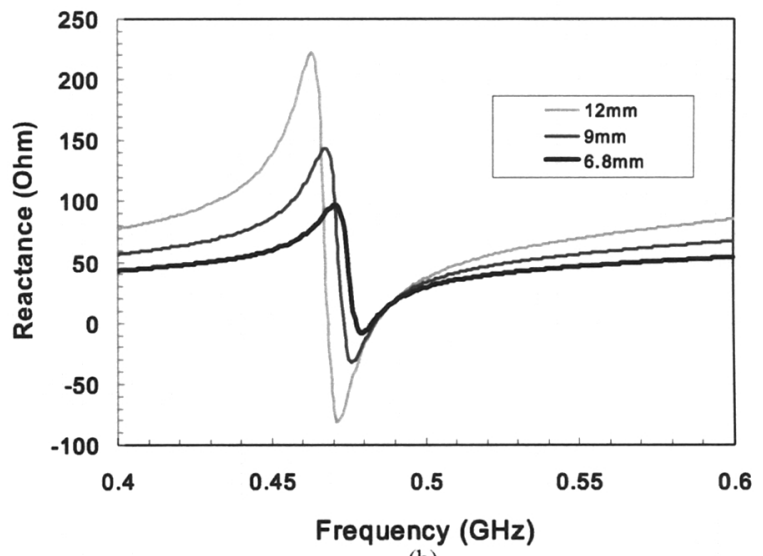

(b)

Fig. 2. Simulated input resistance and reactance of the RFID tag with the shorting stub length of $12 \mathrm{~mm}, 9 \mathrm{~mm}$ and $6.8 \mathrm{~mm}$, respectively. (a) Resistance (b) Reactance.

the area of operation, such as $866-956 \mathrm{MHz}$ in North America/Europe for EPC GEN2 item-level tracking and the lower band around $400 \mathrm{MHz}$ for bio-applications.

One of the main challenges in designing an RFID tag is the impedance matching between the terminals of the tag antenna and those of the IC. This requires a conjugate matching technique, such as series or parallel stubs and/or using inductively coupling. The matching network of the tag has to guarantee the maximum power delivered to the IC which is used to store the data transmitted to and receive from the RFID 


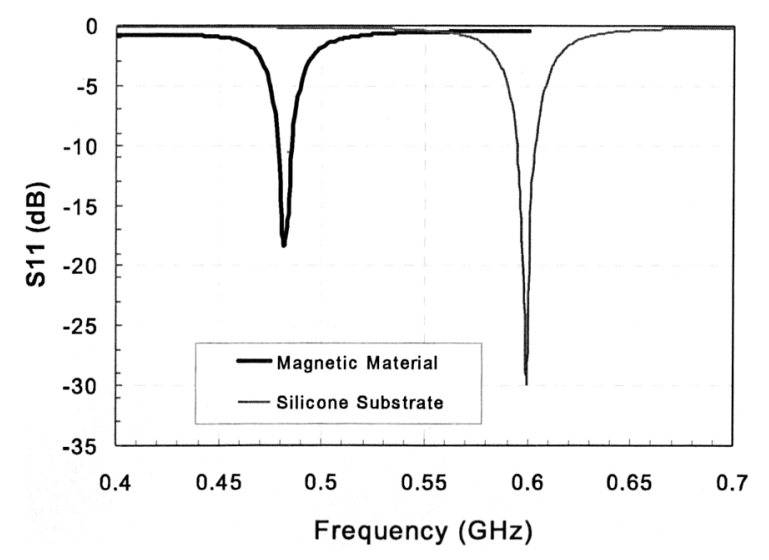

Fig. 3. Measured return loss of the RFID tag antenna on the magnetic material with the comparison of the one on the silicone substrate.

reader. The return loss of RFID antenna can be calculated based on the power reflection coefficient which takes into account the reactance part of the IC's impedance

$$
\left|s^{2}\right|=\left|\frac{Z_{I C}-Z_{A N T} *}{Z_{I C}-Z_{A N T}}\right|^{2}
$$

where $Z_{I C}$ represents the impedance of the IC and $Z_{A N T}$ represents the impedance of the antenna terminals with $\mathrm{Z}_{\mathrm{ANT}}{ }^{*}$ being its conjugate.

Another challenge concerns dimensions of the RFID tags for flexible operation. The free space wavelength at $480 \mathrm{MHz}$ is $692 \mathrm{~mm}$. An RFID tag that has the miniaturized features is becoming more of a necessity, for example, in the implementation of an RFID-enable wristband for wireless health monitoring in hospital.

To achieve these design goals, a folded bow-tie meander line dipole antenna was designed and fabricated on the characterized magnetic composite material substrate. The RFID prototype structure is shown in Fig. 1 along with dimensions, with the IC placed in the center of the shorting stub arm. The nature of the bow-tie shape of the halfwavelength dipole antenna body allows for a broader band operation. The meander line helps realizing further miniaturization of the antenna structure. The shorting stub arm is responsible for the matching of the impedance of the antenna terminals to that of the IC through the fine tuning of the length. Fig. 2 shows the resistance and reactance versus frequency when the shorting stub arm is tuned at $12 \mathrm{~mm}, 9 \mathrm{~mm}$ and $6.8 \mathrm{~mm}$, respectively.

In measurement, a GS $1000 \mu \mathrm{m}$ pitch probe was used for impedance measurements. In order to minimize backside reflections of this type of antenna, the fabricated antenna was placed on a custom-made probe station using high density polystyrene foam with low relative permittivity of value 1.06
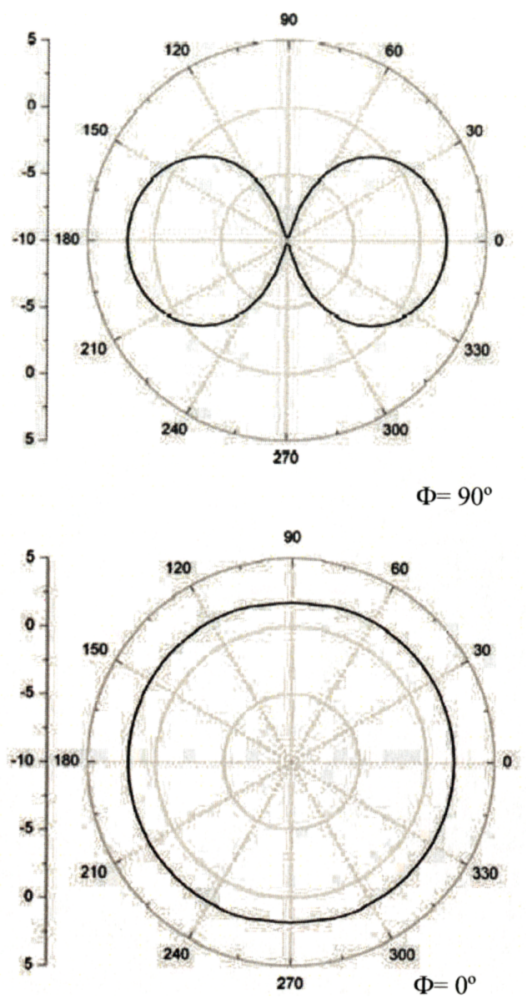

Fig. 4. Simulated 2D far-field radiation pattern plots. An omnidirectional radiation pattern can be observed at $\Phi=0^{\circ}$ plane with directivity around $2.0 \mathrm{dBi}$.

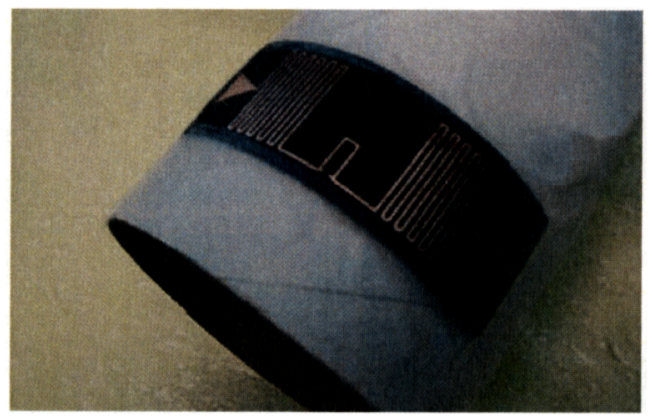

Fig. 5. Photograph of the conformal RFID tag on a foam cylinder.

resembling that of the free space. The calibration method used was short-open-load-thru (SOLT).

The initial structure was designed for the lower end of the UHF spectrum and was modeled using Zeland IE3D full wave EM software. The initial substrate was pure silicone $\left(\varepsilon_{\mathrm{r}}=2.65\right.$ and $\tan \delta=0.001$ ) of $1.3 \mathrm{~mm}$ thickness. Then the same dimensions of the antenna were maintained for the magnetic composite material. The Return Loss plot is shown in Fig. 3, demonstrating a frequency down shifting of $20 \%$ with increased magnetic permeability, which proves the miniaturization concept. The radiation pattern of the RFID tag 


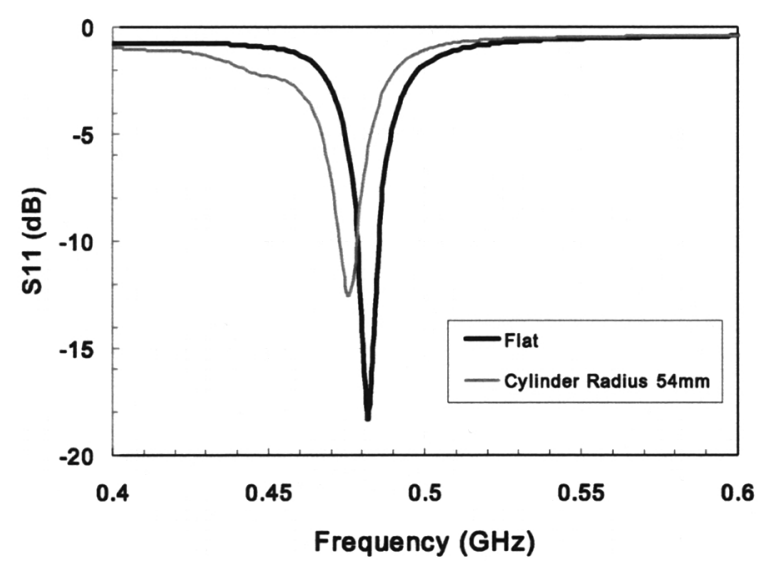

Fig. 6. Measured return loss of the flat RFID tag and the conformal RFID tag. $6 \mathrm{MHz}$ frequency down shifting is observed.

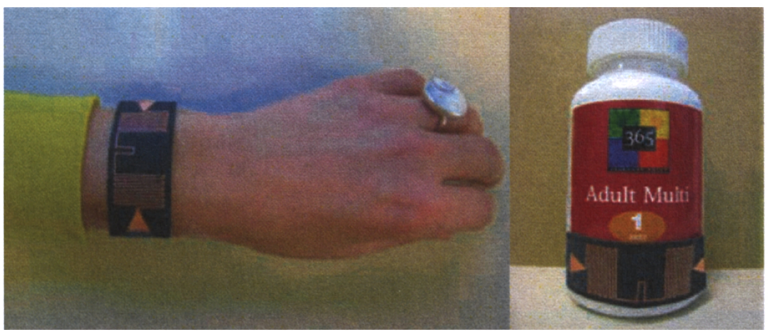

Fig. 7. Embodiments of the conformal RFID tag prototype in the applications of wireless health monitoring and pharmaceutical drug bottle tracking.

module was plotted in Fig. 4. The radiation pattern is almost uniform (omnidirectional) at $480 \mathrm{MHz}$ with directivity around $2.0 \mathrm{dBi}$.

In order to verify the performance of the conformal RFID antenna, measurements were performed as well by sticking the same RFID tag on a foam cylinder, as shown in Fig. 5. The radius of the cylinder is $54 \mathrm{~mm}$. The return loss results in Fig. 6 show that return loss of this conformal RFID antenna is slightly shifted down by $6 \mathrm{MHz}$ with a center frequency at $474 \mathrm{MHz}$. Overall a good performance is still remained with the interested band covered. The flexible property of the substrate enables the RFID tag module's application in diverse areas. Fig. 7 demonstrates the conformal RFID tag prototype in the applications of wireless health monitoring and pharmaceutical drug bottle tracking.

\section{CONCLUSIONS}

A combination of electromagnetic tools and measurements has been used to investigate the impact of magnetic composite materials to the miniaturization of RFID antennas considering geometric, material and fabrication parameters. This approach has been applied to the design of a benchmarking conformal RFID tag module and has enabled the assessment of implications that materials have on this design, specifically that the antenna is miniaturized by using the magnetic composite vs. pure silicone. A real composite material has been fabricated and the performance of the miniaturized antenna predicted using the models. This is a demonstration of a flexible magnetic composite proven for the $480 \mathrm{MHz}$ bandwidth with acceptable magnetic losses that makes it usable for small size, lightweight conformal applications like wireless health monitoring in pharmaceuticals, hospital, ambulance and home-based patient care.

\section{ACKNOWLEDGEMENT}

The authors wish to acknowledge the NSF CAREER ECS9984761, the NSF ECS-0313951, the Georgia Electronic Design Center (GEDC). Special thanks to Dr. Michael D. Hill and Barry W. Treadway of Trans-Tech, Inc., in Adamstown, Maryland, and David J. Meyer and Haiying Li of Motorola in Plantation, Florida.

\section{REFERENCES}

[1] "Magnetic Materials for RFID," TechnoForum 2005, TDK, http: / www.tdk.co.jp/tf2005/pdf e/2fo215e.pdf.

[2] N. Das and A. K. Ray, "Magneto Optical Technique for Beam Steering by Ferrite Based Patch Arrays," IEEE Transactions on Antennas and Propagation, vol. 49, no. 8, August (2001); pp. 1239-1241.

[3] S. Morrison, C. Cahill, E. Carpenter, S. Calvin, R. Swaminathan, M. McHenry, V. Harris, "Magnetic and Structural Properties of Nickel Zinc Ferrite Nanoparticles Synthesized at Room Temperature," Journal of Applied Physics, vol. 95, no. 11, June (2004); pp. 6392-6395.

[4] H. Dong, F. Liu, Q. Song, Z.J. Zhang, C. P. Wong, "Magnetic Nanocomposite for High Q Embedded Inductor," IEEE International Symposium and Exhibition on Advance Packaging Materials: Process, Properties, and Interfaces, Atlanta, Georgia, March (2004); pp. 171-174. 